\title{
VOGEL, Jakob, Ein schillerndes Kristall. Eine Wissensgeschichte des Salzes zwischen früher Neuzeit und Moderne
}

Jean-Claude Hocquet

\section{OpenEdition}

Édition électronique

URL : http://journals.openedition.org/ifha/1789

DOI : $10.4000 /$ ifha. 1789

ISSN : 2198-8943

\section{Éditeur}

IFRA - Institut franco-allemand (sciences historiques et sociales)

\section{Référence électronique}

Jean-Claude Hocquet, «VOGEL, Jakob, Ein schillerndes Kristall. Eine Wissensgeschichte des Salzes zwischen früher Neuzeit und Moderne », Revue de I'IFHA [En ligne], Date de recension, mis en ligne le 01 janvier 2009, consulté le 22 septembre 2020. URL : http://journals.openedition.org/ifha/1789 ; DOI : https://doi.org/10.4000/ifha.1789

Ce document a été généré automatiquement le 22 septembre 2020.

(CIFHA 


\title{
VOGEL, Jakob, Ein schillerndes Kristall. Eine Wissensgeschichte des Salzes zwischen früher Neuzeit und Moderne
}

\author{
Jean-Claude Hocquet
}

1 L'auteur présente son ouvrage comme le fruit d'une fructueuse coopération francoallemande qui s'est fortifiée au sein du Centre Français de l'Université Technique de Berlin (Centre Marc-Bloch) autour de l'équipe réunie par Étienne François qui a favorisé le développement d'un projet de recherche antérieur et lui a conféré une dimension comparatiste. En Allemagne et en Autriche, il a bénéficié de conseils éclairés et d'une richesse archivistique incomparable et en France, à Paris ou Amiens, il s'est inséré dans des équipes qui travaillaient sur « Les Ressources naturelles » et surtout sur « Experts et expertises », ce qu'il me semble important de souligner pour l'élaboration des concepts liés à l'histoire des savoirs et à l'innovation. L'ouvrage fourmille de références à Marc Bloch ou à Michel Foucault et à des historiens français dont les travaux ont nourri une copieuse bibliographie qui occupe 57 pages.

2 J.V. est parti d'une constatation de la vie quotidienne que peuvent faire tous les clients des hypermarchés : il y a peu de temps encore, on achetait un sel de cuisine empaqueté dans un méchant emballage bleu et blanc (les deux couleurs associées au sel, le blanc cristal issu de la mer bleue), aujourd'hui on vous propose du sel aux épices, du sel aux herbes, du sel fumé, du sel spécial pour pizza, du sel ayurvédique, du sel noir extrait des volcans de Hawaï, ou rose et blanc, couleurs alternées des cristaux, originaire de l'Himalaya, et bien entendu du sel iodé aux effets bénéfiques moins sujets à caution. Il est paradoxal de voir que l'âge de la mondialisation (globalisation) et de l'uniformisation des normes culinaires s'ingénie à mettre au point des stratégies de marketing qui diversifient à l'extrême un produit qui est constitué, quand il est pur, du seul chlorure de sodium $(\mathrm{NaCl})$. Cette mutation a pourtant des antécédents que l'auteur recherche dans la richesse et la symbolique du sel à l'époque moderne, dont il voit avec raison le modèle le plus accompli dans la monarchie des Habsbourg et dans les plafonds des salles d'apparat du château de Schönbrunn décorés de poêles et de Salzfuder 
cylindriques. De l'utilisation du sel dans le baptême chrétien à la chimie moderne qui a fait $\mathrm{du}$ sel une matière première indispensable à l'industrie, l'histoire du sel a accompagné tout le progrès industriel, ainsi les techniques de forage minier. Ce qui intéresse le plus l'auteur, c'est l'histoire du savoir scientifique qui s'est développé autour du sel, des premières découvertes et de la modernisation technique des XVIIe et XVIIIe s. à l'industrialisation du XIXe s., de l'apparition d'un nouvelle profession, les experts, et de leur place dans nos sociétés modernes, de l'évaluation d'un processus global de Verwissenchaftlichung de la nature et de la vie quotidienne déjà décrit par Max Weber comme un " désenchantement du monde » (Entzauberung der Welt). On voit l'ambition du projet qui se veut une réflexion sur l'innovation et par conséquent sur la modernisation, deux concepts au cœur de l'industrialisation.

Le livre est découpé en cinq chapitres. Le premier examine à travers un cas de microhistoire quelles difficultés a rencontrées dans les années 1760-1780 un médecin thermal de Karlsbad (Karlovy Vary), David Becher, tenant de la science éclairée, pour imposer l'image nouvelle du sel, produit chimique et sanitaire, à ses collègues de la communauté scientifique, la perspective s'élargissant à un débat entre l'expertise médicale et les contemporains demeurés attachés aux représentations encore diffusées par l'alchimie au milieu du siècle. Le second chapitre conduit en une centaine de pages l'historien du sel en terrain plus familier, celui du développement technique qui suscita des conflits à la fin du siècle entre les connaisseurs du salinage et les experts miniers autour de la question du développement des compétences professionnelles à l'intérieur des salines prussiennes et autrichiennes, l'enjeu portant sur l'adoption du savoir minier et de ses méthodes dans la production du sel (rappelons que dans ces deux régions d'Europe centrale, le sel est un produit minier) pour le développement des nouveaux gisements découverts au début du siècle suivant. Le chapitre suivant revient à la médecine et à la découverte, à Schönebeck par le Dr Tolberg, médecin de la saline, des vertus du thermalisme pour la santé, aux relations qui unissent la santé, la chimie et la technique dans la nouvelle pratique médicale et dans le développement du thermalisme à l'orée du XIXe s. Bref, à l'aide des représentations de l'influence médicale du sel de cuisine, les problèmes de la médecine du temps et de la santé du corps débouchent sur de nouvelles pratiques thérapeutiques. Le chapitre 4 examine la création de la chimie et son application à l'agriculture et la mise en exploitation des gisements de sel de potasse de Stassfurt dans les années 1860 pour la production d'engrais minéral qui a préparé à l'agriculture industrielle triomphante du second XXe s. Dans ce champ nouveau de la recherche s'opposent le découvreur Justus Liebig, auteur précisément de l'ouvrage Die Chemie in ihrer Anwendung auf Agricultur und Physiologie (1840 et nombreuses rééditions successives) et les tenants de la tradition en Allemagne, en France et en Angleterre. Reste alors à examiner quelle influence a exercée le nouveau discours scientifique sur le sel dans les représentations du temps et dans son statut matériel, concrètement la production de sel de cuisine (Kochsalz, les Français disent plutôt « sel de table ») a-t-elle connu des améliorations qualitatives à la fin du XIXe s. ? Un sel plus pur a-t-il succédé au sel artisanal et les producteurs ont-ils répondu aux souhaits des consommateurs ? La réponse est difficile car on ne dispose le plus souvent que des réponses des producteurs qui vantent les qualités et la bonté de leur sel. Quant aux consommateurs, ils témoignent d'un traditionalisme qui refuse les nouveautés en matière de sel, même baptisées " améliorations » et visant à une plus grande pureté chimique, ce dont pourrait témoigner le succès commercial du sel de Guérande en France comme en Allemagne. Le " cristal scintillant » continue d'offrir 
une belle résistance aux tenants de l'alimentation scientifique. Le livre, comme on le voit, se veut une réflexion à la fois historique et philosophique sur le cheminement de la connaissance scientifique et le sel lui offrait une belle illustration : voilà un nouvel usage pour un produit aux multiples facettes qui a illustré l'écart qui peut séparer savoir scientifique et vie quotidienne. Le livre devrait aussi susciter l'intérêt de tous ceux qui aujourd'hui portent intérêt aux questions soulevées par le consumérisme.

4 Jean-Claude HOCQUET (Institut de Recherches Historiques, Université Charles-deGaulle Lille III) 